\title{
Left heart side calcification: coralliform mitral annular calcification and porcelain aorta
}

\author{
Calcificación del corazón izquierdo: anillo mitral coraliforme y aorta en porcelana
}

Luis A. Moreno-Ruiz' ${ }^{1 *}$, Moisés Jiménez-Santos ${ }^{2}$, Noé Zamorano-Velázquez ${ }^{3}$, Marianna Garcia-Saldivia1, Yatzil Necoechea-Osuna ${ }^{1}$, Cristo Kúsulas-Zerón ${ }^{1}$, Jesús Campos-Larios', Agustina Moreno-González ${ }^{1}$, Jessica Bocardo-Galván ${ }^{1}$, Enrique Martínez-Flores ${ }^{4}$, and Luis E. Santos-Martínez ${ }^{5}$

${ }^{1}$ Department of Valvular Heart Disease; ${ }^{2}$ Department of Computed Tomography; ${ }^{3}$ Department of Echocardiography; ${ }^{4}$ Department of Electrophysiology; ${ }^{5}$ Department of Pulmonary Hypertension Clinic and Right Ventricle. Hospital de Cardiología, Centro Médico Nacional Siglo XXI, Instituto Mexicano del Seguro Social (IMSS), Mexico City, Mexico

We present the case of a 70-year-old woman with coralliform mitral annular calcification (MAC) and porcelain aorta with multimodality approach of the mitral disease as shown in Figure 1. The heart team considered inoperable because of high risk of perioperative atrioventricular groove disruption or frank rupture with massive bleeding because of the coralliform MAC and porcelain aorta and consequently death and considered only medical treatment (Society of Thoracic Surgeons of $6.15 \%$ and EuroSCORE II of $6.61 \%$ ). The patient develops nodal rhythm and electrophysiologist decided to install dual-chamber permanent pacemakers. At a 6-month follow-up visit, with optimal medical treatment, she had only mild dyspnea (New York Heart Association Class II). The mitral valve disease is an extreme form of MAC, with extension of calcification into the leaflets and subvalvular apparatus like this case. The association of MAC and porcelain aorta represents great difficulty for surgical treatment because of unsafe aortic cross-clamping or arterial cannulation ${ }^{1}$. Actually, the therapeutic options are multiple in patients with MAC; the Mount Sinai algorithm starts with surgical risk assessment and then anatomical analysis and includes surgical treatment (replace or surgical mitral valve repair), percutaneous or hybrid, and medical treatment in inoperable cases, like our patient ${ }^{2}$.

\section{Funding}

This research has not received any funding from public, commercial, or non-profit agencies.

\section{Conflicts of interest}

None. license (http://creativecommons.org/licenses/by-nc-nd/4.0/).

Available online: 12-04-2021 Arch Cardiol Mex (Eng). 2021;91(2):242-243 www.archivoscardiologia.com 


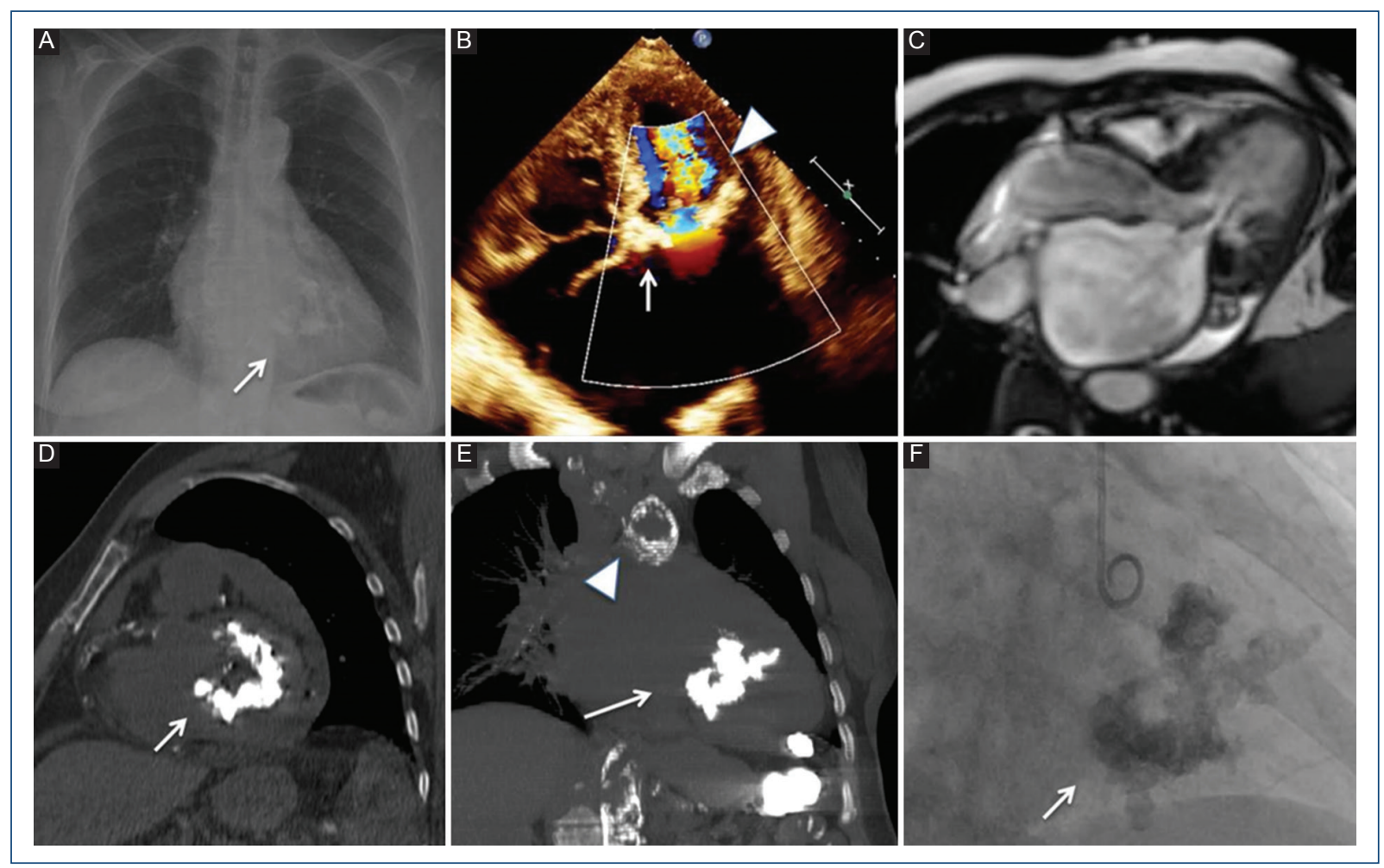

Figure 1. Multimodality imaging of severe coralliform mitral stenosis and porcelain aorta. A: X-ray chest posteroanterior view showed cardiomegaly, mitral annular calcification (MAC), and aorta (arrow). B: echocardiogram apical 4 chamber view confirmed the MAC (arrow), left atrium enlargement, and anterograde accelerated flow through the stenotic mitral valve (head arrow). C: magnetic resonance 4 chamber plane showed mitral stenosis and mild regurgitation. D, E: noncontrast thoracic computed tomography images showed maximum intensity projection reconstructions of a short axis D and two chamber $\mathbf{E}$ views where severe coralliform mitral calcification is shown (arrow), also severe calcification of the aortic arch is depicted (head arrow). F: fluoroscopic image of coralliform MAC (arrow). Note the similarities of the mitral calcification shown by different methods.

\section{Ethical disclosures}

Protection of human and animal subjects. The authors declare that no experiments were performed on humans or animals for this study.

Confidentiality of data. The authors declare that they have followed the protocols of their work center on the publication of patient data.

Right to privacy and informed consent. The authors have obtained the written informed consent of the patients or subjects mentioned in the article. The corresponding author is in possession of this document.

\section{References}

1. Shamsudeen I, Fei LY, Burwash IG, Beauchesne L, Chan V, Glineur D, et al. Presentation and management of calcific mitral valve disease. Int J Cardiol. 2020;304:135-7.

2. El-Eshmawi A, Alexis SL, Sengupta A, Pandis D, Rimsukcharoenchai C, Adams $\mathrm{DH}$, et al. Surgical management of mitral annular calcification. Curr Opin Cardiol. 2020;35:107-15. 